\title{
Towards a model of full-sky Galactic synchrotron intensity and linear polarisation: A re-analysis of the Parkes data
}

\author{
G. Giardino ${ }^{1}$, A. J. Banday ${ }^{2}$, K. M. Górski ${ }^{3,4}$, K. Bennett ${ }^{1}$, J. L. Jonas ${ }^{5}$, and J. Tauber ${ }^{1}$ \\ 1 Research and Science Support Department of ESA, ESTEC, Postbus 299, 2200 AG Noordwijk, \\ The Netherlands \\ 2 Max-Planck Institut für Astrophysik, Garching bei München, 85741, Germany \\ 3 ESO, Garching bei München, 85748, Germany \\ 4 Warsaw University Observatory, Warsaw, Poland \\ 5 Department of Physics \& Electronics, Rhodes University, PO Box 94, Grahamstown 6140, South Africa
}

Received 21 December 2001 / Accepted 22 February 2002

\begin{abstract}
We have analysed the angular power spectra of the Parkes radio continuum and polarisation survey of the Southern galactic plane at 2.4 GHz. We have found that in the multipole range $l=40-250$ the angular power spectrum of the polarised intensity is well described by a power-law spectrum with fitted spectral index $\alpha_{L}=$ $2.37 \pm 0.21$. In the same multipole range the angular power spectra of the $E$ and $B$ components of the polarised signal are significantly flatter, with fitted spectral indices respectively of $\alpha_{E}=1.57 \pm 0.12$ and $\alpha_{B}=1.45 \pm 0.12$. Temperature fluctuations in the $E$ and $B$ components are mostly determined by variations in polarisation angle. We have combined these results with other data from available radio surveys in order to produce a full-sky toy model of Galactic synchrotron intensity and linear polarisation at high frequencies $(\nu \gtrsim 10 \mathrm{GHz})$. This can be used to study the feasibility of measuring the Cosmic Microwave Background polarisation with forthcoming experiments and satellite missions.
\end{abstract}

Key words. radio continuum: ISM - surveys - polarization - cosmic microwave background

\section{Introduction}

Current cosmological models predict the level of polarisation of the Cosmic Microwave Background (CMB) to be at $5-10 \%$ of the temperature anisotropies at the $20 \mathrm{arcmin}$ angular scale, that is at $\mu \mathrm{K}$ level (e.g. Bond \& Efstathiou 1987). These temperature variations have to be detected over a $3 \mathrm{~K}$ continuum with instrumentation whose typical noise temperature is of the order of tens of Kelvin. The detection of the CMB polarisation represents therefore a considerable challenge. To date, only upper limits on the CMB polarisation exist. The most recent results from ground based observations place an upper limit of $10 \mu \mathrm{K}$ on any polarised signal from the sky at the $7^{\circ}$ angular scale in the frequency band $26-36 \mathrm{GHz}$ (Keating et al. 2001) and an upper limit of $15 \mu \mathrm{K}$ at the $1^{\circ}-0.5$ angular scale at $90 \mathrm{GHz}$ (Hedman et al. 2001).

Current cosmological models also predict a shape for the angular power spectra of the polarised component of the CMB, so the measure of these spectra will provide additional information on the properties of the primordial density fluctuations and on the thermal history of the universe (e.g. Kosowsky 1996). The variation of

Send offprint requests to: G. Giardino,

e-mail: ggiardin@rssd.esa.int polarised CMB emission is predicted to peak at sub-degree angular scale. The NASA satellite mission MAP will have enough sensitivity to detect the CMB polarisation, if this is present at the $\mu \mathrm{K}$ level (Kogut \& Hinshaw 2000). The future ESA mission Planck will have higher sensitivity and may provide direct imaging of the CMB polarised signal (Mandolesi \& Puget 1998). The future SPORT experiment that is planned to be placed on the International Space Station also has the sensitivity to detect $\mu \mathrm{K}$-level polarised signal from the CMB if this is present at angular scales $\gtrsim 7^{\circ}$ (Fabbri et al. 1999).

A fundamental question for any measurement of the polarisation of the CMB is whether the expected signal can be distinguished from the foreground polarised signal from our galaxy. The interstellar magnetic field is illuminated by cosmic-ray electrons which spiral around field lines and thereby emit synchrotron radiation. This radiation is intrinsically highly linearly polarised, $70-75 \%$ in a completely regular field (e.g. Rybicki \& Lightman 1979). Thermal emission from dust may also be highly polarised, depending on the shape and alignment of the dust particles (Wright 1987; Prunet et al. 1998).

As in the case of the unpolarised emission, knowledge of the angular power spectra of the foreground polarised component is essential when trying to recover the 
angular power spectra of the CMB. In this paper we use the Parkes $2.4 \mathrm{GHz}$ polarimetric survey of the southern Galactic plane in order to derive the angular power spectra of the polarised component of the Galactic synchrotron emission. The survey has relatively small sky coverage but has 10 arcmin angular resolution, which allows the statistical properties of polarised synchrotron emission to be investigated at the angular scales where the CMB polarised signal is expected to peak.

In order to assess the feasibility of CMB polarisation measurements, study the effect of instrumental systematics and devise the data analysis strategy it is necessary to perform simulations of the satellite missions. For these simulations an input sky with all the known components of the emission is necessary. By exploiting existing observations of the microwave sky, we have to strive to construct a simulated sky which represents our best guess of some of the properties of the real sky in the unexplored regions and frequency channels. This will allow us to perform more realistic simulations of the mission and to be in a better position to interpret the real data when these become available.

We use the results of our analysis of the Parkes survey, combined with other data on synchrotron emission coming from total intensity large sky radio surveys, to produce a full-sky synthetic map of synchrotron polarised emission. The paper is organised as follows. In Sect. 2 the global angular power spectra of the Parkes $2.4 \mathrm{GHz}$ polarimetric survey are derived. The simulations performed to assess the reliability of the spectra are presented in Sect. 3. The results are discussed in Sect. 4. In Sect. 5 the derived spectra are used as basis to construct a toy model of the all-sky polarised synchrotron emission.

\section{The Parkes data at $2.4 \mathrm{GHz}$}

The Parkes $2.4 \mathrm{GHz}$ survey is a sensitive, polarimetric survey of the southern Galactic plane carried out by Dancan et al. (1997). The survey covers $127^{\circ}$ of Galactic longitude $\left(238^{\circ} \leq l \leq 5^{\circ}\right)$, with a latitude coverage out to at least $\pm 5^{\circ}$ (up to $b=7^{\circ}$ and $b=8^{\circ}$ over some longitudes). The angular resolution of the images is $10.4 \mathrm{arcmin}$. The nominal rms noise of the survey (for the total and polarised intensities) is $5.3 \mathrm{mK}$. The survey is publicly available and the data come split into 6 rectangular fields sampled at a pixel resolution of 4 arcmin. The linear polarisation signal is given in terms of the two Stokes parameters $Q$ and $U$.

The Parkes survey has been recently analysed by Baccigalupi et al. (2001) and Tucci et al. (2000). In both of these previous studies, the data were split into twelve square patches, each of size $10^{\circ} \times 10^{\circ}$, which were then individually analysed. Our work differs from these in that the power spectra of the different polarisation components are derived from a global analysis of the complete Parkes data.

In order to perform this global statistical analysis of the survey we have resampled the six fields of the original data into HEALPix tessellations. The iso-Latitude property of the HEALPix tessellation of the sphere makes it ideal for fast computation of the angular power spectrum of a field defined on the sphere (Górski et al. 1999). In the HEALPix tessellation the angular size of the pixels is determined by the nside parameter. We used a HEALPix tessellation with nside $=1024$ which corresponds to pixels of linear size 3.4 arcmin. When resampling the survey, the pixel value in the HEALPix tessellation was assigned by weighted integration of the values of the original pixels that overlapped with the new pixel, where the weight of each pixel contribution is the fraction of its area intersecting with the new pixel. This approach ensures that no spurious pixel-to-pixel signal correlation is introduced by the resampling.

The three channels of the survey $(T, Q$ and $U)$, resampled into HEALPix are shown in Fig. 1. The derived intensity of linear polaristion, $L=\sqrt{Q^{2}+U^{2}}$, is also shown. The maps are in Galactic coordinates.

The most striking difference between the total intensity channel $(T)$ and the polarisation channels $(Q, U$ and $L$ ) is that the total intensity signal is dominated by the signal from the Galactic plane while in the polarisation channels the Galactic plane is hardly visible. Moreover many point sources which are clearly visible in the total intensity channel do not exhibit a polarised counterpart.

In the polarised intensity image the most apparent features are the extended areas of polarisation, with sizes of the order of $5^{\circ}$ or so, which do not appear to be connected with discrete sources of total power emission. Such regions lie around longitudes $265^{\circ}, 280^{\circ}, 320^{\circ}$ to $330^{\circ}$ and $355^{\circ}$ to $5^{\circ}$. While one of these structures can be identified with the Vela supernova remnant at $l=265^{\circ}$, the origin and nature of the other features is not yet explained (Duncan et al. 1997).

Five regions that are bright in the $T$ maps have been blanked out to prevent a significant fraction of instrumental polarisation from being introduced. These are Sgr A and the bright H II complexes G353.2+0.8, G284.14-0.3, G287.5-0.6 and G291.28-0.71.

\subsection{Derivation of the power spectra}

The three Stokes parameters $T, Q$ and $U$ fully describe any state of linearly polarised light. However, whereas the temperature is a scalar quantity (invariant under rotation of the plane of the sky), $Q$ and $U$ are not. They depend on the direction of observation $\hat{n}$ and on the Cartesian axes perpendicular to $\hat{n}$ used to define them. Recent theoretical developments have shown that it is more useful to describe the polarisation field in terms of two quantities that are invariant under rotation (Zaldarriaga \& Seljak 1997; Kamionkowski 1997). These two quantities are usually called $E$ and $B$ and they are obtained by a linear, non-local, transformation of the $Q$ and $U$ map (hereafter we will refer to this transformation as to the $E-B$ transform). The $E$ and $B$ components differ in their behavior under parity transformation: $B$ changes sign while $E$ does 

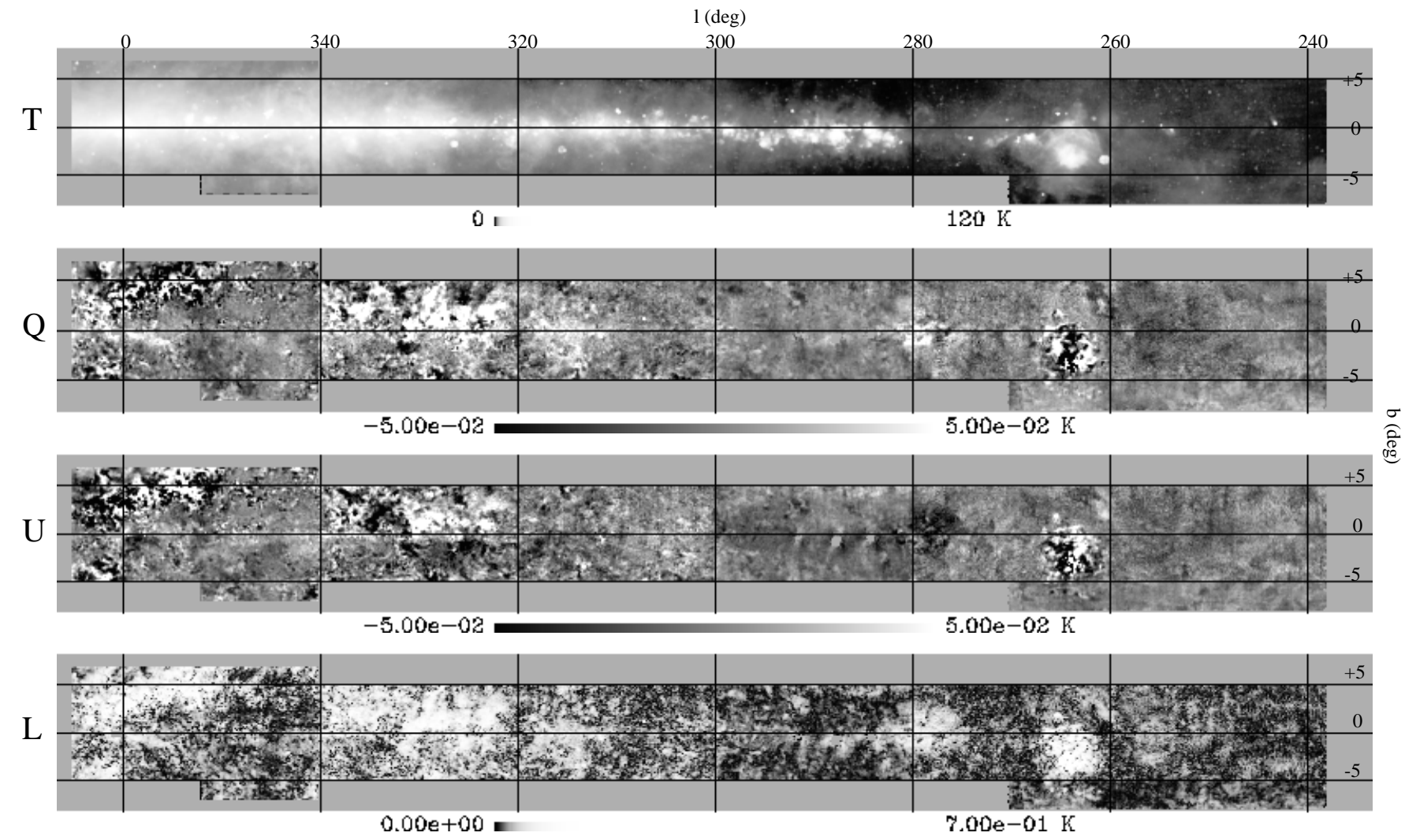

Fig. 1. The Parkes survey at $2400 \mathrm{MHz}$. The six fields of the original data have been resampled into one HEALPix tessellation with a pixel size of 3.4 arcmin. The first three images (from the top) give the three Stokes parameters of the signal: $T, Q, U$, respectively; the lowest image shows the derived polarised intensity $L=\sqrt{Q^{2}+U^{2}}$. The temperature scales for $T$ and $L$ have been histogram equalised.

not. Four power spectra are needed to characterise the fluctuations in a Gaussian theory: the autocorrelation of each of $T, E$ and $B$ and the cross correlation between $E$ and $T$.

Current cosmological models predict the CMB intensity and polarisation variations to be a Gaussian field and therefore give predictions for the four power spectra $T, E$, $B$ and $E \times T$. Galactic diffuse emission is not a Gaussian field and so cannot be fully characterised by the four power spectra, however, since we are interested in comparing the statistical properties of this emission with predictions for the CMB power spectra we have derived the four power spectra of $T, E, B$ and $E \times T$ of the Parkes data.

The power spectra have been obtained by performing a spin-weighted harmonic analysis of the field in the HEALPix grid with the ANAFAST program (the software is part of the HEALPix package). The results of the analysis for the whole set of data are summarised in the following subsections. The angular power spectra of the polarised intensity was derived by simple spherical harmonic decomposition, also using the ANAFAST program.

From the analysis of large sky surveys, the angular power spectra of the various components of unpolarised Galactic foreground emission appears to be well described by power law spectra with a spectral index between 2 and 3. Below, we model the angular power spectra of the different components of the Parkes data with power-laws of the form:

$C_{l}^{X}=k l^{-\alpha_{X}}$

where $l$ is the multipole order and $X$ may stand for $T, L$, $E, B, e$ and $b$, with $e$ and $b$ indicating the $E-B$ transform of the sinus and cosinus of the polarisation angle.

The limited sky coverage of the survey and the FWHM resolution of 10.4 arcmin limit our spectral analysis to the multipole range $l=40-250$. Section 3 describes Monte Carlo simulations that were made to assess the effects of reduced sky coverage and finite resolution.

\subsubsection{Total and polarised intensity}

The angular power spectrum of the $T$ channel of the Parkes survey is shown in Fig. 2. In the multipole range $l=40-250$ a linear least-squares fit to this spectrum gives: $\alpha_{T}=1.67 \pm 0.15^{1}$. In the figure the angular power spectrum of the intensity of the polarised signal $L$ is also shown. Linear least-squares fit to this spectrum gives $\alpha_{L}=2.37 \pm 0.21$.

\footnotetext{
${ }^{1}$ Errors quoted in this paper are estimated via Monte Carlo simulations unless otherwise stated.
} 


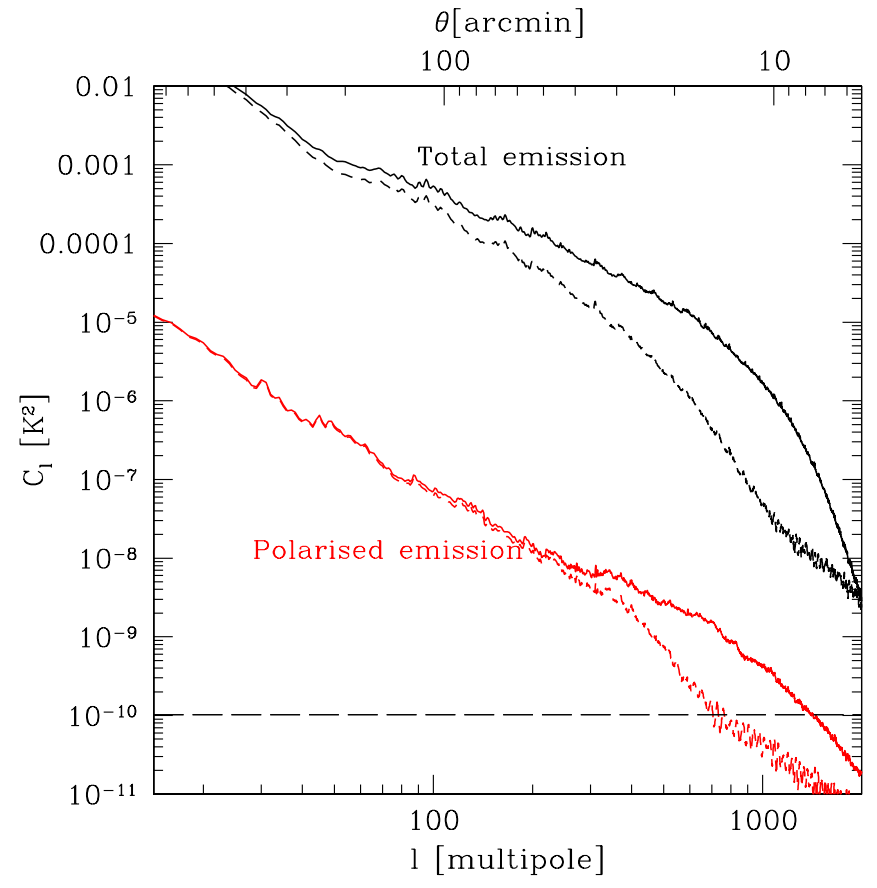

Fig. 2. The global angular power spectra of $T$ and $L=$ $\sqrt{Q^{2}+U^{2}}$ for the Parkes survey (continuous lines). The power spectra of $T$ and $L$ after median filtering are also shown (dashed lines). Point source suppression by the filtering process is responsible for the steepening of the intensity spectrum. Such steepening is not observed in the spectrum of polarised emission (up to $l \sim 150$ ). The horizontal line gives the nominal noise level. The multipole order $l$ corresponds to a typical angular scale of $\theta=\pi / l$.

From the images in Fig. 1 point sources are clearly identifiable in the map of total emission while they are not identifiable in the polarised channels. In order to verify whether point sources or noise at pixel scale is affecting the spectra derived from the Parkes survey, we median filtered the intensity map $(T)$ and the derived polarised intensity map $(L)$ by convolving the data with a kernel of $9 \times 9$ pixels. We have used this technique to derive the angular power spectrum of diffuse emission from the large sky radio survey of Jonas et al. (1998). We showed that median filtering can be used to effectively remove point sources and derive the angular power spectrum of the diffuse component (Giardino et al. 2001).

The angular power spectrum of a population of point sources randomly distributed in the sky is Poissonian, that is with constant power at all $l$. Therefore, if point sources contribute significantly to the temperature fluctuation in the field of sky, the power spectrum of that field is expected to steepen when the point sources are removed and only the diffuse components remain. The same happens if the signal is dominated by instrumental random noise. On the other hand if the signal in the field is dominated by the diffuse component, median filtering will not have any effects on the spectrum of the emission up to the multipole order where the high-frequency fluctuations of the emission are averaged out. We estimated by Monte Carlo simulation that, for a beam with a $F W H M$ of 10.4 arcmin and a map with pixel size of 3.4 arcmin, median filtering with a $9 \times 9$ pixel kernel allows the input angular power spectrum of a diffuse component (with a spectral index of $\alpha=2$ ) to be derived up to $l=150$ with an error lower than $3 \%$.

The results of the analysis of the median filtered $T$ and $L$ maps are also shown in Fig. 2.

From the figure it is apparent that the spectrum of the total emission steepens significantly upon the application of the filtering process. The fitted spectral index values, in the $l$ range $40-150$, before and after median filtering are respectively $\alpha_{I}=1.68 \pm 0.03$ and $\alpha_{I}^{\mathrm{mf}}=2.05 \pm 0.03$ (only the formal fit errors are given in this case $\left.{ }^{2}\right)$. This indicates that a significant fraction of the power spectrum at intermediate and high $l$ is due to the presence of discrete signals: either a population of point sources or instrumental noise at the pixel scale. In fact instrumental noise at pixel scale is unlikely as the nominal noise level is well below the spectrum derived for the total intensity. We therefore interpret the steepening observed as due to the removal of the point source contribution.

Figure 2 shows no comparable change in the slope (for $l<150$ ) of the angular power spectrum of the derived $L$ map after median filtering. The fitted spectral index values before and after median filter are respectively $\alpha_{L}=$ $2.34 \pm 0.03$ and $\alpha_{L}^{\mathrm{mf}}=2.45 \pm 0.03$.

This analysis implies that, up to multipole order $l=$ 150 , the fluctuations in the polarised emission are of diffuse nature and they are not due to the presence of discrete signals such us point sources or instrumental noise at pixel scale.

Even though we can exclude contamination of the polarisation spectrum by point sources and instrumental noise at pixel scale only up to multipole $l=150$, in the following sections we derive the spectral indices for the other components of the Parkes data over the entire multipole range $l=40-250$ that can be directly probed given the survey's sky coverage and angular resolution. The reader should note from Fig. 2 that the slope of the power spectrum of the unfiltered polarised emission does not obviously change between an $l$ of 150 and 250, and therefore the fits given up to 250 should also be consistent with those made only up to 150 . Indeed, we have explicitly established this.

\subsubsection{The $E$ and $B$ components}

The power spectra of the $E$ and $B$ components derived from the Parkes survey are shown in Fig. 3. In the multipole range $l=40-250$ linear least-squares fits to the spectra give $\alpha_{E}=1.57 \pm 0.12$ and $\alpha_{B}=1.45 \pm 0.12$ respectively for $E$ and $B$. As can be seen from the figure the power spectra of the $E$ and $B$ components are very similar

\footnotetext{
2 Since in this case we are interested in the spectral indices' relative values before and after the filtering, the formal fit errors are representative of the uncertainty in this comparison.
} 


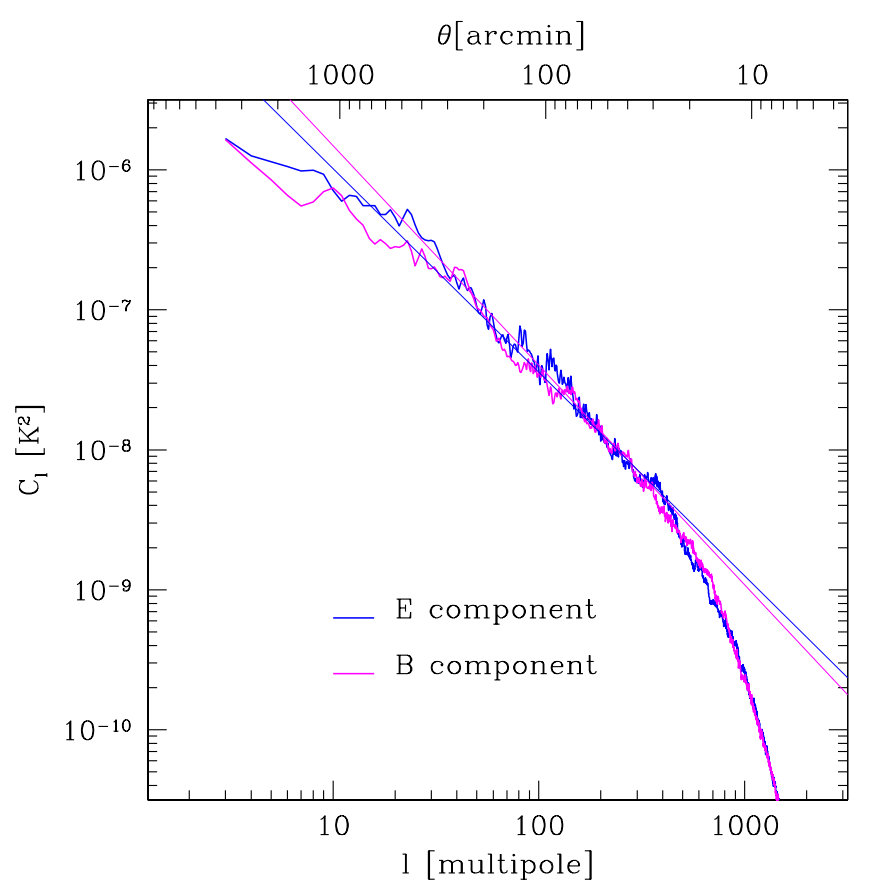

Fig. 3. The power spectra of the $E$ and $B$ components for the Parkes data. The linear least squares fits to the spectra in the multipole range 40-250 are also shown.

in slope and amplitude. This is expected if the observed emission field does not show clear patterns or symmetries (Zaldarriaga 2001).

\subsubsection{The $E \times T$ component}

The cross-spectrum of the $E$ and $T$ components is shown in Fig. 4.

In order to assess whether the computed correlation signal corresponds to null correlation between $E$ and $T$ we performed a series of simulations. We generated ten random Gaussian polarised fields using the spectral indices and normalisation derived from the Parkes data to define the power spectra of $T, E$ and $B$ and setting $C_{l}^{E \times T}$ to zero. The same sky coverage and beam FWHM of the Parkes survey were reproduced (see Sect. 3).

The $E \times T$ spectra derived from the ten simulations showed a standard deviation around 0 of $\sigma_{l}=1.4 \times$ $10^{-4} / l(l+1) \mathrm{K}^{2}$. The grey shaded region in Fig. 4 shows the area within $\pm 3 \sigma_{l}$. The comparison of the data points with this area is consistent with the absence of correlation between $E$ and $T$. If a level of correlation between $E$ and $T$ exists, this is below the level that can be probed with the Parkes data, given the survey's sky coverage and beam FWHM.

The power spectrum derived from $E$ and the median filtered $T$ is also shown in the figure. The level of correlation is hardly affected by the filtering process (up to the multipole order $l=150$ where the two spectra are directly comparable), implying that the lack of correlation between the $T$ and the $E$ component is not due to the presence of unpolarised point sources (which only affect the $T$ map).

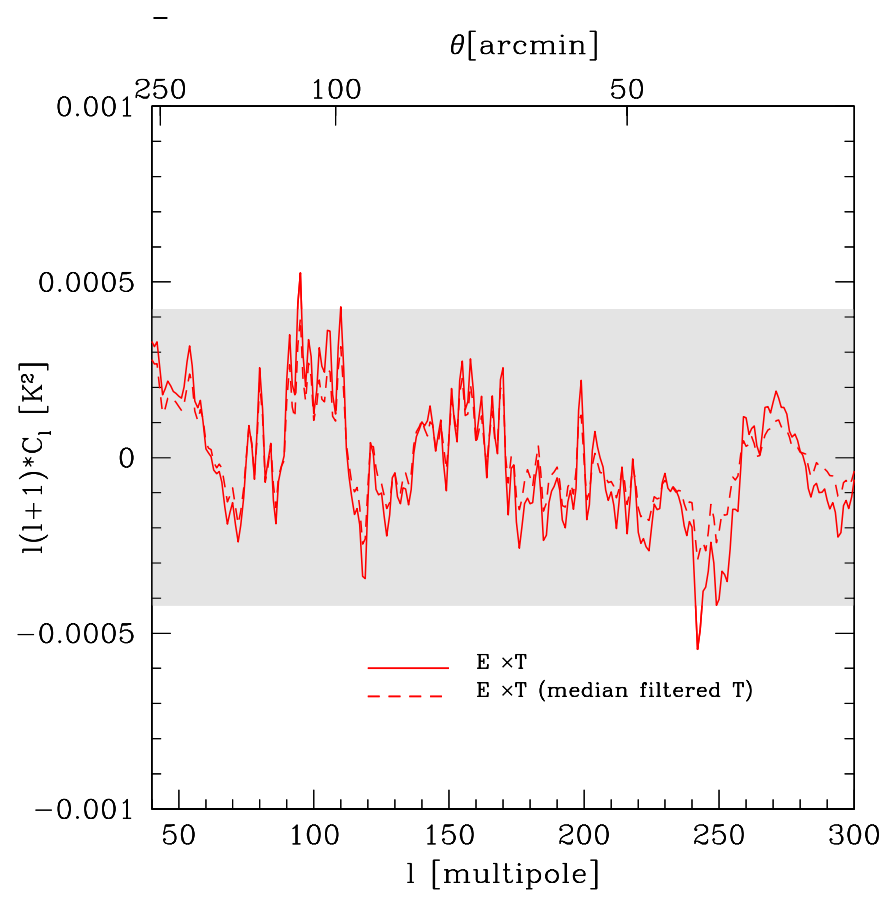

Fig. 4. The cross-correlation $E \times T$ for the Parkes data. The grey area gives $\pm 3 \sigma$ from zero of the recovered $E \times T$ spectra for 10 simulations with input null $E \times T$ (see text).

\subsubsection{The polarisation angle}

In order to quantify the statistical properties of the polarisation angle variations we constructed the maps of $\cos (2 \theta)$ and $\sin (2 \theta)$, where $\theta$ is the polarisation angle. These maps are simply given by $Q / L$ and $U / L$ respectively. The sinus and cosinus maps were then $E-B$ transformed to obtain the rotationally invariant quantities, $e$ and $b$. The power spectra of $e$ and $b$ were also derived by spin-weighted harmonic analysis. The result of the analysis is shown in Fig. 5.

In the multipole range $l=40-250$, the power spectra of $e$ and $b$ are well described by power law spectra with fitted spectral indices of $\alpha_{e}=1.74 \pm 0.14$ and $\alpha_{b}=1.69 \pm 0.13$ respectively. These values are very close to the spectral indices of the power spectra of the $E$ and $B$ components.

\section{Error assessment}

In order to verify the reliability of the power spectra derived from the Parkes survey we performed a series of Monte Carlo simulations. We generated 10 full-sky realisations of a random Gaussian polarised field defined by four power spectra with shape and normalisation similar to the ones of the Parkes data. For $T, E$ and $B$ we used powerlaw spectra with spectral indices of $\alpha_{T}=1.7, \alpha_{E}=1.5$, $\alpha_{B}=1.5$ respectively, for $E \times T$ the spectrum was set to zero. The 10 full-sky random realisations of these spectra were convolved with a Gaussian beam with a FWHM of 10.4 arcmin and sampled using a HEALPix tessellation with $n$ side $=1024$. The power spectra for each realisation were then derived by performing spin-weighted harmonic 


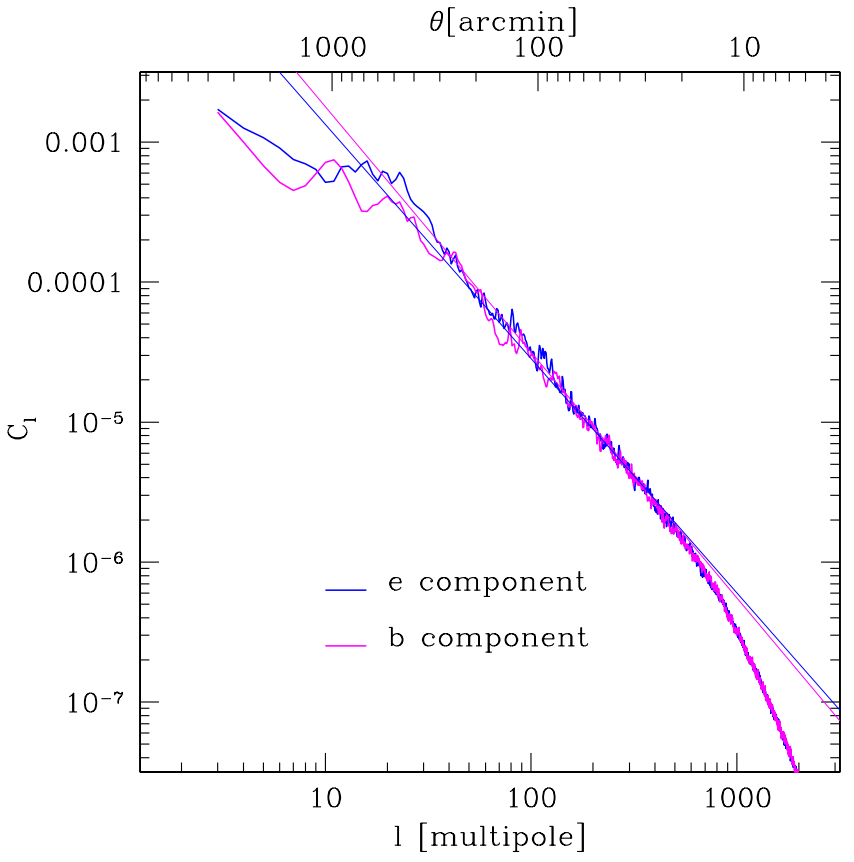

Fig. 5. The power spectrum of the E-B transform of $\cos (2 \theta)$ and $\sin (2 \theta)$, where $\theta$ is the polarisation angle. The transformed map are here indicated with $e$ and $b$. The linear least squares fits to the spectra in the multipole range $l=40-250$ are also shown.

analyses for both full-sky coverage and the limited Parkes sky coverage.

The averaged power spectra from the 10 simulations are shown in Fig. 6. The top panels shows the derived average power spectra of $T$ and the bottom panels the derived average power spectra of $E$ and $B$. The two different cases of sky coverage are shown. The effect of the incomplete sky coverage is visible in the spectra in the right panels where the average of the derived spectra deviates significantly from the input spectra (the true value) for $l<40$. Above multipole order $l>250$ the derived power spectra are affected by the beam cut-off.

In order to quantify the accuracy of the recovery we have computed the best-fit power law of the spectra obtained from the 10 realisations, in the two different cases of sky coverage. The spectral indices of these best-fit power laws can be compared with the true spectral indices of the power spectra used as inputs for the 10 realisations. The standard deviation of the derived spectral indices with respect to the true (input) values provides an estimate of the standard error on the spectral index. The results are summarized in Table 1 . In the case of full sky coverage and considering the multipole range $l=3-250$, one can recover the input spectral indices of $T, E$ and $B$ with an error of approximately $2 \%$ (as can be seen by taking the ratio of the SD over the mean in three columns of the table). The limited sky coverage of the Parkes survey implies that one can only fit a power law to the recovered spectra in the multipole range $l=40-250$. By considering the spectrum in this limited multipole range the error on the recovered spectral indices increases significantly even when using spectra derived from full-sky maps (around $5 \%$, as taken from Table 1 ). The error is around $10 \%$ when recovering the indices of the $T, E$ and $B$ spectra from maps with the limited sky coverage of the Parkes survey.

\section{Comparison with previous results and discussion}

The spectral index of $\alpha_{T}=1.67 \pm 0.15$ that we derived from the Parkes data for the total intensity $(T)$ is significantly lower than the spectral index values derived from large sky radio survey (Bouchet \& Gispert 1999; Giardino et al. 2001). However this value refers to the raw data which beside the diffuse emission contains a significant population of point sources. After point source removal the index steepens to $\alpha_{T}=2.05 \pm 0.15$. This value can be compared with the value of $\alpha_{T}=1.9$ for the spectral index of the same region of the sky of the Rhodes/HartRAO survey after point source removal.

The spectral index of the Rhodes/HartRAO survey steepens with Galactic latitude from $\alpha=2.4$ when all the data are considered to $\alpha=2.9$ when only the data at galactic latitude $|b|>20^{\circ}$ are taken into account (Giardino et al. 2001). Therefore it is not surprising that when a region within Galactic latitude $|b|=8^{\circ}$ is considered the derived spectral index is flatter. The reason for this is probably a combination of the contributions from supernovae remnants and from the high concentration of diffuse H II emission regions in the Galactic plane.

As earlier noted, the Parkes survey was previously analysed by Baccigalupi et al. (2001) and Tucci et al. (2000). Baccigalupi et al. restricted their analysis to the total intensity and the polarised intensity data $(L)$ while Tucci et al. (2000) present results for the power spectra of $T, E$ and $B$, but not $L$.

Baccigalupi et al. (2001) obtained an average value of $\alpha_{L}=1.7 \pm 0.2$ for the spectral index of the angular power spectrum of the polarised intensity of the twelve patches in the $l$-range $100-800$. This value is significantly lower than the value of $\alpha_{L}=2.37 \pm 0.21$ that we derived for the multipole range $l=40-250$. However, there are two issues to consider:

1. the angular power spectrum of the unfiltered $L$ (Fig. 2) shows a flattening of the slope around $l=300$.

2 . in order to analyse the Parkes data beyond $l \sim 250$ where the beam suppression becomes appreciable, assumptions about the noise amplitude and distribution are necessary.

If we perform a linear least squares fit to $C_{l}^{L}$ in the multipole range $l=100-800$, assuming purely Gaussian random noise and the nominal noise level given in Duncan et al. (1997), we obtain $\alpha_{L, l=100-800}=1.75$, in close agreement with the value derived by Baccigalupi et al. On the other hand, if we take into account the presence of the slope break and fit the curve with two power laws, we 


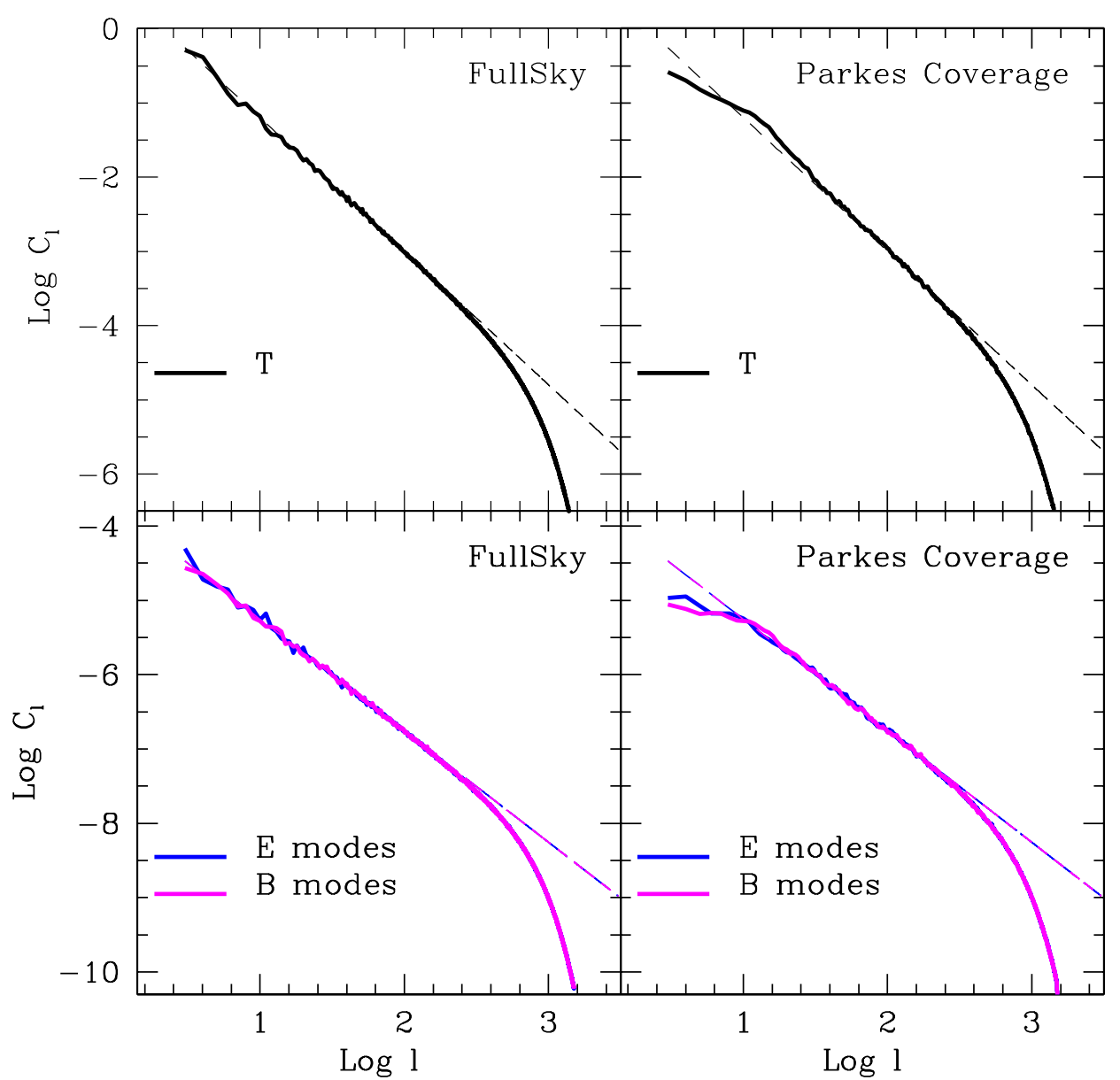

Fig. 6. The average spectra of $T$ (top) and of $E$ and $B$ (bottom) obtained from the spin-weighted harmonic analysis of 10 different realisations of a polarised Gaussian radiation field (continuous lines). The dashed lines indicates the input power spectra for $T, E$ and $B$. These are power law with spectral indices $\alpha_{T}=1.7, \alpha_{E}=1.5, \alpha_{B}=1.5$ (very similar to the Parkes data). The input spectrum for $E \times T$ was set to zero.

Table 1. Mean and standard deviation of the recovered spectral indices from 10 different sky realisations of a random Gaussian field with input spectra with spectral indices $\alpha_{T}=1.7, \alpha_{E}=1.5, \alpha_{B}=1.5$ for $T, E$ and $B$. The spectrum of $E \times T$ was set to zero. Two different cases of sky coverage have been considered: full sky coverage and the Parkes survey sky coverage.

\begin{tabular}{cccccccc}
\hline \hline & & $\alpha_{T}$ & & $\alpha_{E}$ & \multicolumn{3}{c}{$\alpha_{B}$} \\
\hline$l$ range & Sky coverage & mean & SD & mean & SD & mean & SD \\
\hline $3-250$ & Full & 1.70 & 0.02 & 1.51 & 0.03 & 1.51 & 0.03 \\
$40-250$ & Full & 1.76 & 0.08 & 1.56 & 0.08 & 1.55 & 0.08 \\
$40-250$ & Parkes & 1.78 & 0.15 & 1.57 & 0.12 & 1.55 & 0.12 \\
\hline
\end{tabular}

obtain $\alpha_{L, l=40-250}=2.35$ and $\alpha_{L, l=250-800}=1.64$, indicating a flattening of the spectrum at $l>250$.

Clearly, Baccigalupi et al. derived a flatter spectral index by fitting the spectrum of $L$ in the l-range 100-800 without accounting for the slope break. As to the reason why their analysis did not see evidence for a change in slope of the power spectrum of $L$, it is likely that by analysing small patches, the derived individual spectra are noisier, thus rendering it more difficult to detect such a change.

Unfortunately, assessing the significance of this flattening is difficult precisely because the analysis for $l>250$ is sensitive to assumptions about the noise amplitude and its distribution.

Tucci et al. (2000) derived the average angular power spectra of the $E$ and $B$ components for the twelve patches and concluded that in the multipole range $36 \leq l \lesssim 1000$ they are both well approximated by power laws with spectral index $\alpha \simeq 1.4$. This value is very similar to the values of $\alpha_{E}=1.57 \pm 0.12$ and $\alpha_{B}=1.45 \pm 0.12$ that we derived for the $E$ and $B$ components respectively.

The angular power spectrum of $e$ and $b$, the $E-B$ transform of the sinus and cosinus of the polarisation angle, were first derived in the present work. In the multipole 
range $l=40-250$ the power spectra of the $e$ and $b$ components of the Parkes data are well described by power laws with fitted spectral indices of $\alpha_{e}=1.74 \pm 0.14$ and $\alpha_{b}=1.69 \pm 0.13$. These values are very close to the spectral indices derived for the $E$ and $B$ components. Fluctuations in $e$ and $b$ are only determined by variations in polarisation angle. Therefore this analysis shows that fluctuations in the $E$ and $B$ components are mostly determined by variations in the polarisation angle rather than polarisation intensity, which, over this multipole range, is characterised by a significantly steeper power spectrum.

Finally, we did not find evidence of correlation between $E$ and $T$ in the Parkes data. This lack of correlation can be interpreted as a direct consequence of the fact that fluctuations in the $E$ components are mostly determined by changes in the polarisation angle, which $T$ remains unaffected by.

Since we are interested in using these results to construct a toy model of the full-sky synchrotron polarisation at high frequencies $(30-100 \mathrm{GHz})$, there are two fundamental questions that we need to ask.

i) What is the effect of Faraday rotation on the Parkes data?

As a result of propagation through an ionized medium, the direction of linear polarisation is rotated by an angle proportional to the radiation wavelength: $\phi=R M \lambda^{2}$.

Gaensler et al. (2001) have made high resolution (1 arcmin) polarimetric observation of a $28 \mathrm{deg}^{2}$ region in the Parkes field $(325.5 \leq l \leq 332.5,-0.5 \leq b \leq 3.5)$ at $1.4 \mathrm{GHz}$. For this region, they derive a mean $R M$ over the entire region of $-12.9 \pm 0.1 \mathrm{rad} \mathrm{m}^{-2}$. This at $2.4 \mathrm{GHz}$ corresponds to approximately $11^{\circ}$. However, the $R M$ values span from 10 to $1000 \mathrm{rad} \mathrm{m}^{-2}$, with typical uncertainties in $R M$ measurements of $\pm 20-40 \mathrm{rad} \mathrm{m}^{-2}$. At a frequency of $2.4 \mathrm{GHz}$ and resolution of 10.4 arcmin, a linear gradient of $5 \mathrm{rad} \mathrm{m}^{-2} \operatorname{arcmin}^{-1}$ in a foreground screen can significantly depolarise background radiation (Sokoloff et al. 1998) and affect the measured variation of polarisation angle. Beam depolarisation is also significant at this frequency and resolution for a level of dispersion in $R M$ of $30 \mathrm{rad} \mathrm{m}^{-2}$ (e.g. Tribble 1991).

Gaensler et al. (2001) conclude that the brightest polarised features seen in this direction of the Galactic plane most likely represent intrinsic structure in the source of emission, but fainter structures are best explained as being imposed by Faraday rotation on a uniformly polarised background by foreground material.

This is likely to be the case also at $2.4 \mathrm{GHz}$ and implies that a significant fraction of the angular variations which determine the power spectra of $E$ and $B$ for the Parkes data are likely to be induced by Faraday rotation. This scenario would predict that the angular power spectra of $E$, and $B$ of synchrotron emission derived from the Parkes survey may be flatter than if they were derived from observations at frequencies higher than $10 \mathrm{GHz}$, where Faraday effects are negligible (Beck 2001).

ii) How representative are the results here derived on the angular power spectrum of linearly polarised emission at low Galactic latitudes of what may happen at high Galactic latitudes?

Duncan et al. (1997) show that over much of the longitude range covered by the survey, there exists a background polarised component which appears to be independent of latitude (over the range of latitudes covered by the survey). Besides the Parkes data, Baccigalupi et al. (2001) also analysed five $10^{\circ}$-patches from the Northern galactic plane survey by Duncan et al. (1999) at $2.7 \mathrm{GHz}$ and three patches of different sizes at medium Galactic latitudes $(2.5 \leq b \leq 17.5)$ from the survey of Uyanıker et al. (1999) at $1.4 \mathrm{GHz}$. By averaging the derived spectral indices for the polarised emission from all twenty fields they obtain $\alpha_{L, l=100-800}=1.8 \pm 0.3$. They concluded that synchrotron polarisation maintains essentially the same statistical properties up to Galactic latitude $b \sim 10^{\circ}$.

However, by comparing high Galactic latitude fields with observations of the Galactic plane at $1.4 \mathrm{GHz}$ Gray et al. (1999) find that the magneto-ionic medium which induces the Faraday rotation on polarised emission appears to be concentrated in the disc component of the Galaxy $\left(b \lesssim 10^{\circ}\right)$. This would imply that higher latititude observations of synchrotron polarised emission at low frequencies and for similar angular resolution may yield steeper angular power spectra.

Indeed, if there is a variation of the slope with Galactic latitude one may expect this change to be in line with what is observed for the total emission, for which the angular power spectrum steepens with Galactic latitude. Moreover, as pointed out by Davies \& Wilkinson (1999), the magnetic field pattern may be more ordered at high Galactic latitudes, resulting in less small-scale structure.

\section{Full-sky synthetic maps of synchrotron polarisation}

As a starting point for creating a toy model of Galactic synchrotron polarisation at high frequencies we assume that the polarised component of synchrotron emission in a given direction is proportional to the unpolarised intensity, which in this case is expressed in terms of brightness temperature, $T$ :

$Q=f T \cos (2 \theta)$

$U=f T \sin (2 \theta)$

where $f$ is the fraction of polarised emission (or polarisation degree) and $\theta$ is the polarisation angle. As we have seen, at the frequency of Parkes observations this is clearly not a valid assumption, but it can be an acceptable starting point at frequencies higher than $10 \mathrm{GHz}$, where Faraday effects (of rotation and depolarisation) may be neglected.

The brightness temperature spectrum of synchrotron emission is well described by a power law,

$T(\nu) \propto \nu^{-\beta}$

where $\beta$ is referred to as the frequency spectral index. In the absence of non-uniform magnetic fields, the fraction 
of polarised radiation $f$ is related to the spectral index (Cortiglioni \& Spoelstra 1995):

$f=\frac{3 \beta-3}{3 \beta-1}$.

Therefore in order to generate synthetic full-sky polarisation maps of synchrotron emission at a given frequency, we need at least three basic ingredients: a full-sky map of the total synchrotron emission at a "template" frequency, a full-sky map of the frequency spectral index and a model for the polarisation angle. The full-sky map of the spectral index will be used to generate $f$ in a given direction and to extrapolate the total emission "template" to the other frequencies where $Q$ and $U$ are generated according to Eq. (1).

\subsection{Synchrotron total intensity $(T)$}

The 408-MHz survey of Haslam et al. (1982) provides, up to date, the best image of the total intensity of the fullsky Galactic synchrotron emission. At this low frequency the synchrotron emission dominates the Galactic diffuse signal (Beuermann et al. 1985; Lawson et al. 1987). We used the $408-\mathrm{MHz}$ survey processed by D. P. Finkbeiner, M. Davis and D. Schlegel (private communication). They have removed the point sources and de-striped the map by applying a Fourier filtering technique. Hereafter we will refer to this processed version of the $408-\mathrm{MHz}$ survey as to the Cleaned 408-MHz map.

The angular resolution of the $408-\mathrm{MHz}$ survey is $0.85^{\circ}$. The angular resolution of Planck will vary from the 33 arcmin $F W H M$ in the $30 \mathrm{GHz}$ channel to 5 arcmin FWHM in the $857 \mathrm{GHz}$ channel. Since it is conservative to assume that synchrotron emission will present structure also at the smaller angular scale, we have superimposed artificial fluctuations at angular scale $\lesssim 1^{\circ}$ on the original map.

The map of artificial fluctuations was obtained from a full-sky realisation of a Gaussian field having a power law spectrum with index $\alpha=3$ for $l>150$ and vanishing to zero for $l<150$. Before being added onto the Cleaned $408 \mathrm{MHz}^{3}$ the fluctuation map is weighted by the Cleaned $408 \mathrm{MHz}$ map itself ${ }^{4}$. This operation can be viewed as an extrapolation of the spectrum of the Cleaned $408 \mathrm{MHz}$ map beyond $l=150$ with a power law spectrum with index $\alpha=3$. A power law spectrum with spectral index $\alpha=3$ is similar to that derived for the total synchrotron emission

\footnotetext{
${ }^{3}$ Convolved with a beam with $F W H M$ of 0.9 .

4 A Gaussian realisation of a power law spectrum with index $\alpha=3$, for $l>150$ and vanishing to zero for $l<150$ has (by definition) power spectra with the same amplitude for all regions of the map. Such a map of artificial fluctuations (let us call it $F$ ) cannot be added onto the Cleaned $408 \mathrm{MHz}$ (let us called this map $H)$, where fluctuation amplitude for $(l<150)$ is a strong function of the position of the sky. By multiplying $F$ with $H / \max (H)$ ("weighting") one obtains a map of fluctuations (at $l>150$ ) for which the amplitude varies as a function of their position in a manner consistent with the Haslam map.
}

at high galactic latitude $\left(|b|>20^{\circ}\right)$ from the analysis of the three large sky radio survey up to $l \sim 100$ (Bouchet \& Gispert 1999; Giardino et al. 2001).

The Cleaned $408 \mathrm{MHz}$ map with extrapolated fluctuations provides us with a realistic image of the full-sky total intensity of synchrotron emission at $408 \mathrm{MHz}$ up to the degree angular scale and an estimate of how this emission may look like at the smaller unobserved angular scales. We use this map as the template for the Stokes $T$ channel of the synthetic polarisation maps. The template at $408 \mathrm{MHz}$ is shown in Fig. 7.

\subsection{Synchrotron frequency spectral index}

Besides the 408-MHz survey the other two high quality, large sky radio surveys available to date are: the northern sky survey of Reich \& Reich (1986) at $1420 \mathrm{MHz}$ and the more recent southern sky survey of Jonas et al. (1998). The sky coverage of the $1420-\mathrm{MHz}$ survey and of the $2326-\mathrm{MHz}$ survey are complementary to each other. The two surveys overlap over the Galactic declination range $-20^{\circ}<\delta<32^{\circ}$ in the right ascension interval $180^{\circ} \leq \mathrm{RA} \leq 360^{\circ}$ and over Galactic declination range $-20^{\circ}<\delta<13^{\circ}$ in the right ascension interval $0^{\circ}<\mathrm{RA}<180^{\circ}$. Therefore we can combine the $408-\mathrm{MHz}$ survey and the $1420-\mathrm{MHz}$ survey in order to derive a map of the spectral index of radio emission in the northern sky and the $408 \mathrm{MHz}$ and the $2326 \mathrm{MHz}$ survey in order to derive a map of the spectral index in the southern hemisphere. We obtained three homogeneous maps in the following way: we took the surveys, as they are publicly available, resampled them into a HEALPix tessellation with nside $=256$ and then median filtered with a box kernel of $9 \times 9$ pixels in order to suppress the point source signal.

As we wish to determine the spectral index of the Galactic emission only, the extragalactic emission must first be subtracted. This is made up of two isotropic components, the CMB and the sum of unresolved extragalactic background. The CMB brightness temperature is $2.73 \mathrm{~K}$. A phenomenological expression to estimate the integrated brightness temperature of extra galactic unresolved radio sources at different frequencies is given by Lawson et al. (1987). Lawson uses it to estimate this contribution in the $408 \mathrm{MHz}$ and in the $1420 \mathrm{MHz}$ surveys. We have adopted it to evaluate this contribution at $2326 \mathrm{MHz}$. The total amount of extragalactic contribution for the three surveys together with basic information about their uncertainties is summarised in Table 2. For the $1420 \mathrm{MHz}$ survey we adopted a baseline correction of $-0.13 \mathrm{~K}$ (Lawson et al. 1987)

After subtraction of the extragalactic contribution, the brightness temperature frequency spectral index $\beta_{\nu 1 / \nu 2}$ of the Galactic radiation can be calculated. It is simply given by:

$\beta_{\nu_{1} / \nu_{2}}=-\frac{T_{\nu_{1}} / T_{\nu_{2}}}{\log \left(\nu_{1} / \nu_{2}\right)}$ 


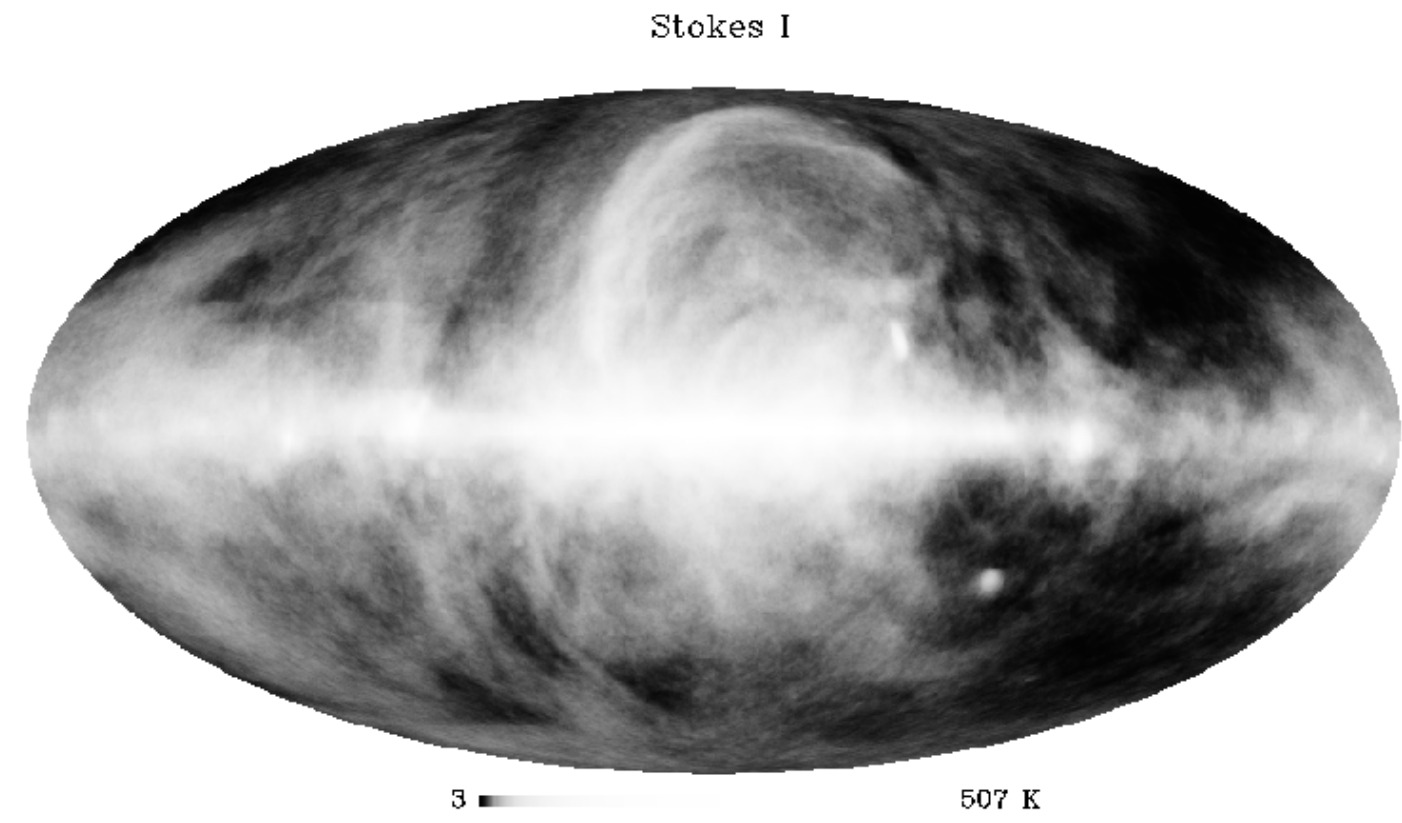

Fig. 7. Mollweide projection of the Cleaned $408 \mathrm{MHz}$ map with extrapolated fluctuations (i.e. the template for the Stokes parameter $T$ of the synthetic polarisation maps). The map is in Galactic coordinates. The color scale has been histogram equalised.

Table 2. Characteristics of the three radio continuum surveys that we used to produce a full-sky map of the radio brightness temperature spectral index.

\begin{tabular}{cccccc}
\hline \hline $\begin{array}{c}\text { Survey } \\
\text { Frequency (MHz) }\end{array}$ & $\begin{array}{c}\text { Scale } \\
\text { error (per cent) }\end{array}$ & $\begin{array}{c}\text { Base level } \\
\text { error }(\mathrm{K})\end{array}$ & $\begin{array}{c}\text { Extragalactic } \\
\text { background }(\mathrm{K})\end{array}$ & $\begin{array}{c}\text { Baseline } \\
\text { correction }(\mathrm{K})\end{array}$ & Reference \\
\hline 408 & 5 & 3 & 5.92 & - & Lawson et al. (1987) \\
1420 & 10 & 0.6 & 2.83 & -0.13 & Lawson et al. (1987) \\
2326 & 5 & 0.080 & 2.75 & - & Jonas et al. (1998) \\
\hline
\end{tabular}

Over the whole observed region the average value of the spectral index obtained by combining the $408 \mathrm{MHz}$ and the $1420-\mathrm{MHz}$ survey is $\left\langle\beta_{408 / 1420}\right\rangle=2.78 \pm 0.17$. By combing the $408 \mathrm{MHz}$ and the $2326-\mathrm{MHz}$ survey one obtains an average value of the spectral index of $\left\langle\beta_{408 / 2326}\right\rangle=2.75 \pm 0.12$, over the whole region observed. Here, the error is only the formal rms of the spectral index maps and does not account for the systematic uncertainties. The mean values for $\beta$ agree well with the value found by Platania et al. (1998) of $\left\langle\beta_{408 / 3800}\right\rangle=2.73 \pm 0.08$.

All the surveys are affected by scan-to-scan baseline errors. When the ratio of brightness temperatures at two different frequencies is calculated the effect of these residual errors is enhanced. Because of this, the map of $\beta_{408 / 1420}$ and the map of $\beta_{408 / 2326}$ do not match in the overlapping region. We tried various techniques to look for a baseline offset for one of the three input maps that would minimise the differences between the two spectral index maps. However discontinuities between the two spectral index maps always remained apparent.

In order to construct the full-sky map of the spectral index, therefore, we used $\beta_{408 / 1420}$ for regions of the sky at declination $\delta>13^{\circ}$ and $\beta_{408 / 2326}$ for regions at declina- tion $\delta<-20^{\circ}$. In the overlapping region of the 1420 and $2326-\mathrm{MHz}$ surveys $\left(-20^{\circ} \leq \delta \leq 13^{\circ}\right)$ we set the spectral index to:

$\beta_{\text {overlap }}=g \beta_{408 / 1420}+h \beta_{408 / 2326}$

where $g=\exp \left[-0.5\left(\delta-13^{\circ}\right)^{2} /\left(10^{\circ}\right)^{2}\right]$ and $h=1-g$. This choice of the weights $g$ and $h$ ensures that the two $\beta$-maps merge smoothly into each other. The region of sky at declination $\delta<83^{\circ}$ remains un-observed at $2326 \mathrm{MHz}$. So we "padded" this region with the spectral index value $\left\langle\beta_{408 / 2326}\right\rangle$.

The spectral index map obtained in this way is however still affected by the striation due to the scan-to-scan baseline errors in the input data. It is possible to remove the effect by Fourier filtering the data but, because the scanning direction differs from one survey to the other, one would not then be able to combine like with like. Following Lawson et al. (1987) we adopted the simpler procedure of convolving the data to a lower resolution, so that the baseline errors are averaged sufficiently for the striation on the color-scale to disappear. The final map of the spectral index is at a resolution of $10^{\circ}$ (at FWHM), as shown in Fig. 8. 


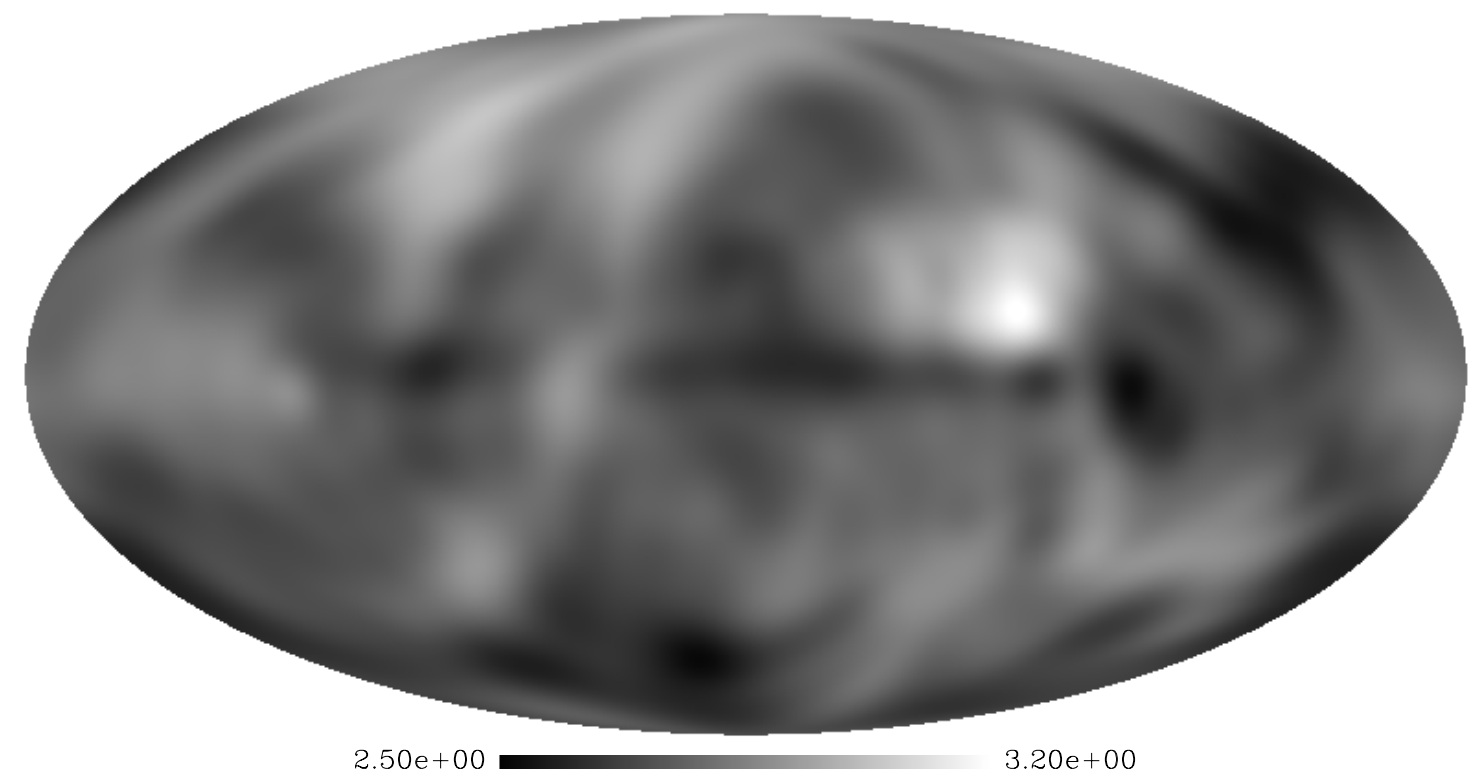

Fig. 8. Mollweide projection of the full-sky map of radio spectral index obtained by combining the 408-MHz survey, the $1420-\mathrm{MHz}$ survey and the $2326-\mathrm{MHz}$ survey (see text). The map is in Galactic coordinates.

\subsection{Synthetic polarisation angle}

We found that the power spectra of the $E-B$ transform of the sinus and cosinus of the polarisation angle of the Parkes data are described by power laws with spectral indices of $\alpha_{e}=1.74 \pm 0.14$ and $\alpha_{b}=1.69 \pm 0.12$ in the multipole range $l=40-250$. One can construct a full-sky map of a random angle having $E-B$ transform of its sinus and cosinus with the same spectra of the $e$ and $b$ component of the Parkes data in the following way. Generate two full-sky realisations of a Gaussian field characterised by a power law spectrum with spectral index $\alpha=1.7$. Let us call these maps $x$ and $y$. Normalise $x$ and $y$ to the interval $[-1,1]$. Generate the full-sky map of the random angle by computing $\theta=\frac{1}{2} \arctan (x / n, y / n)$, where $n=\sqrt{x^{2}+y^{2}}$. We used the random angle map obtained in this way as the polarisation angle of our toy model of Galactic synchrotron emission.

Figure 9 shows the map of the polarisation angle derived from the Parkes data and the map of the synthetic polarisation angle for the same region of sky. The structure of the angle fluctuations in the two maps looks similar.

\subsection{The synthetic maps at 30 and $100 \mathrm{GHz}$}

In order to generate the synthetic maps of synchrotron emission and linear polarisation at higher frequencies the total intensity map at $408 \mathrm{MHz}$ has to be extrapolated. One can use the spectral index map directly, assuming a fixed power law to higher frequencies, or use it in conjunction with the local electron spectrum, as in Bennett et al. (1992), in order to derive maps of $\beta$ between any two given frequencies and to attempt to allow for the observed steepening of $\beta$ with increasing frequency (Lawson et al. 1987; Banday \& Wolfendale 1990).
For the local electron spectrum, we used the polynomial fit to the spectral shape of electron measurements given in Bennett et al. (1992), for energies $0.1<$ $E(\mathrm{GeV})<100$, and likewise a power law $E^{-3.312} \mathrm{~d} E$ for $E>100 \mathrm{GeV}$. By interpreting the spatial variation in $\beta(408-1420 \mathrm{MHz}$ and $408-2326 \mathrm{MHz})$ as due solely to variations in a synchrotron effective Galactic magnetic field, $B_{\text {eff }}$, we derived a full-sky map ${ }^{5}$ of $B_{\text {eff }}$, with $\left\langle B_{\text {eff }}\right\rangle=2.0 \pm 2.3 \mu \mathrm{G}$ and a median value of $1.4 \mu \mathrm{G}$. This is remarkably close to the values derived by Bennett et al. (1992) who did not have the data from the 2326-MHz survey for the southern hemisphere.

The same expression for the local electron spectrum and the map of synchrotron effective Galactic magnetic field were then used to obtain the maps of the spectral index between $408 \mathrm{MHz}$ and 30 and $100 \mathrm{GHz}$, necessary for extrapolating the total emission map. The $\beta$ maps mean values are $\left\langle\beta_{0.408 / 30}\right\rangle=2.91 \pm 0.09$ and $\left\langle\beta_{0.408 / 100}\right\rangle=2.96 \pm 0.08$. The value of $\left\langle\beta_{0.408 / 30}\right\rangle$ is consistent with the upper limit of $\beta>3$ between $408 \mathrm{MHz}$ and $31.5 \mathrm{GHz}$ obtained by Kogut et al. (1996) and the value of $\beta \sim 2.8$ between $1420 \mathrm{MHz}$ and $19 \mathrm{GHz}$ derived by de Oliveira-Costa et al. (1998).

The $Q$ and $U$ channels of the synthetic linear polarisation maps of Galactic synchrotron emission at 30 and $100 \mathrm{GHz}$ were then obtained by combining the intensity map $(T)$ extrapolated at 30 and $100 \mathrm{GHz}$ with the corresponding spectral index map and the random angle map, according to Eqs. (1) and (3). The $Q$ and $U$ channels at $30 \mathrm{GHz}$ are shown in Fig. 10. The three input maps are sampled into a HEALPix tessellation with a pixel size of 6.9 arcmin $($ nside $=512$ ). Therefore also the synthetic

\footnotetext{
${ }^{5}$ Nearly full-sky: only the region at declination $\delta<83^{\circ}$ remains un-observed at $2326 \mathrm{MHz}$.
} 


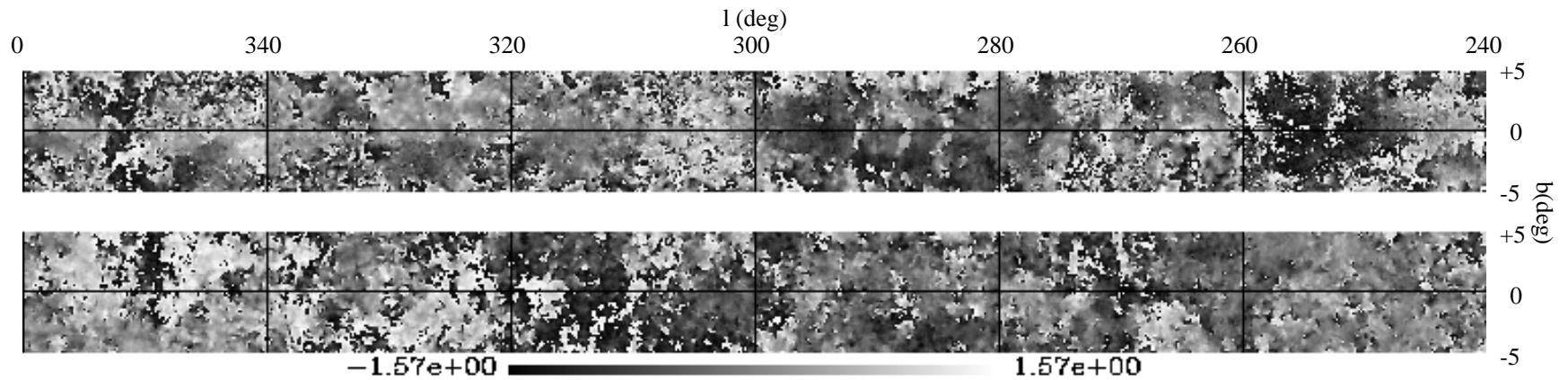

Fig. 9. Polarisation angle from the Parkes survey (top) and from the synthetic map of synchrotron polarisation (bottom). Maps are in Galactic coordinates.
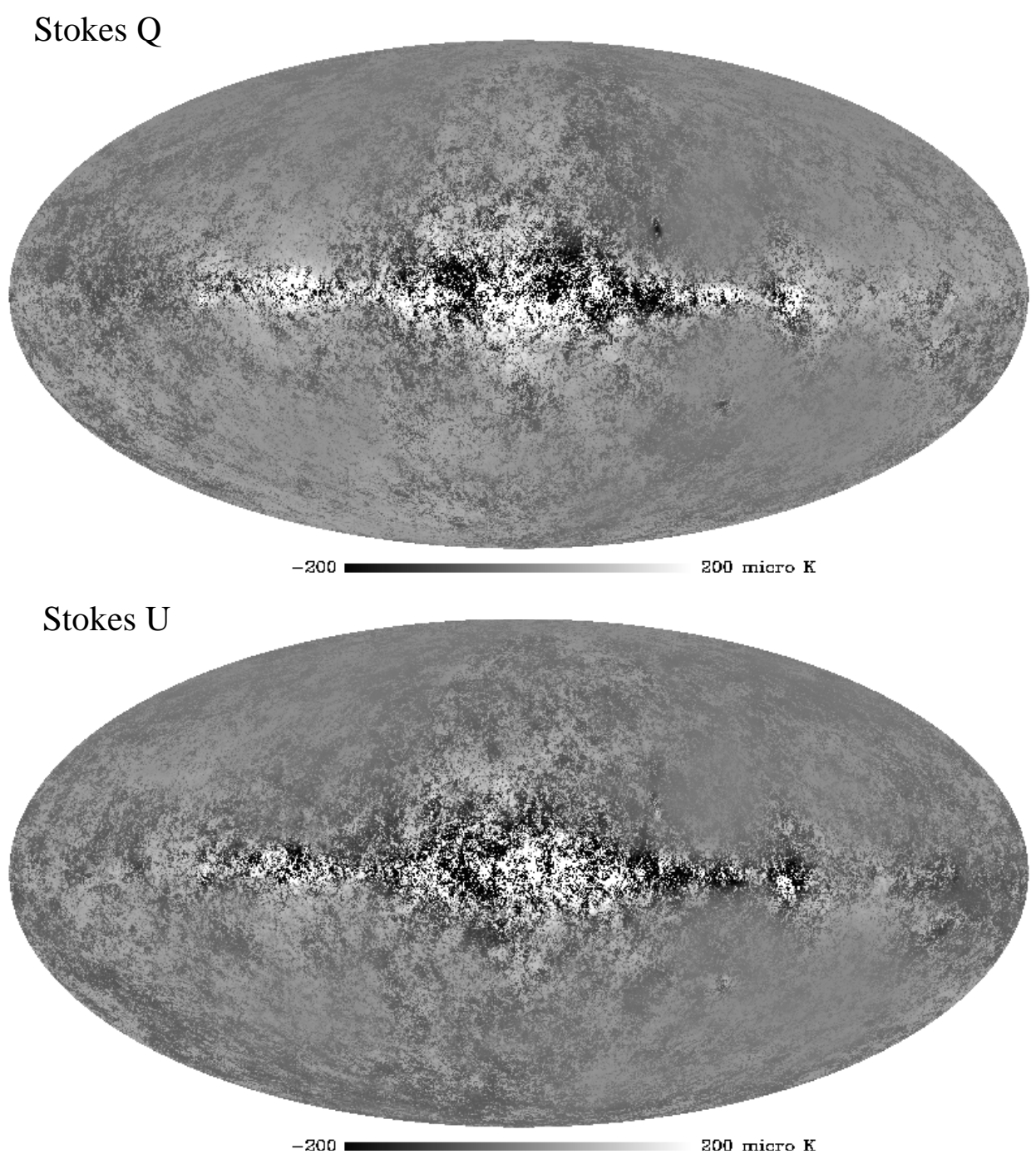

Fig. 10. The $Q$ and $U$ channel of the synthetic map of Galactic synchrotron linear polarisation at $30 \mathrm{GHz}$. The map is in Galactic coordinates.

maps of $T, Q$ and $U$ are at a pixel resolution of 6.9 arcmin.

Because our toy model for $f$ is a very slowly varying function of the position in the sky the maps of $Q$ and $U$ derived according to Eq. (1) have temperature fluctuations mostly determined by the polarisation angle. Therefore, like for the Parkes data, the angular power spectra of the
$E$ and $B$ components of the synthetic linear polarisation maps reflect the spectra of $e$ and $b$. They are power-law spectra with indices $\alpha_{E}=1.67 \pm 0.09$ and $\alpha_{B}=1.73 \pm 0.09$ over the $l$ range $40-250\left(\alpha_{E}=1.59 \pm 0.03\right.$ and $\alpha_{B}=$ $1.68 \pm 0.03$ for the $l$ range $3-250$, that can be probed since in this case the power spectra are derived from full sky maps). These values are very close to the values derived 
for the spectra of the $E$ and $B$ components of the Parkes data.

\subsubsection{Comparison of the power spectra with predictions for the CMB}

For CMB studies one is particularly interested in the properties of radio emission at high Galactic latitudes because this is where the Galactic signal is weaker and where sensitive CMB measurements can be made. Therefore, we computed the angular power spectra of the $T, E$ and $B$ components of the extrapolated maps with a Galactic plane cut-off at latitude $|b|=20^{\circ}$. The results are shown in Fig. 11, where they can be compared with the signal expected from the CMB. The CMB spectrum has been computed assuming a flat inflationary model with purely scalar scale-invariant fluctuations, vacuum energy $\Omega_{\Lambda}=0.70$, cold dark matter density $h^{2} \Omega_{\mathrm{cdm}}=0.12$ and baryon density $h^{2} \Omega_{\mathrm{b}}=0.024$, (where $h=0.7$ is the assumed Hubble parameter), as derived by Netterfield et al. (2001) from combining the latest CMB observations with constraints derived from measurements of the large scale structure and results from recent measurements of type Ia supernovae.

The top panel of Fig. 11 shows the angular power spectra of the $T$ components of the two extrapolated maps and of the CMB. Note the absence of a perceivable change in the slope of the power spectra of the $T$ components of the synthetic maps at $l=150$, the multipole order at which artificial signal was added to the observed signal at $408 \mathrm{MHz}^{6}$.

From the figure, it is apparent that at $100 \mathrm{GHz}$ the contribution to the total sky signal from high latitude synchrotron emission is negligible. At $30 \mathrm{GHz}$ synchrotron emission can be a significant contribution to the sky anisotropy at scales larger than $\sim 20^{\circ}$, but it is more than an order of magnitude weaker than the computed cosmological signal at degree angular scales where the first "acoustic" peak of the CMB is observed. This confirms the results discussed in Giardino et al. (2001) that were obtained with a different approach using the index of the angular power spectra of the survey at $2326 \mathrm{MHz}$ by Jonas et al. (1998) and the COBE DMR upper limit to synchrotron temperature fluctuations at $31.5 \mathrm{GHz}$ at the $7^{\circ}$ scale. The COBE DMR upper limit (Kogut et al. 1996) is indicated in the figure by a pentagonal point.

The angular power spectra of the $E$ and $B$ components of the synthetic maps are shown in the lower panel of Fig. 11. At small angular scale the power of temperature fluctuations in the $E$ and $B$ components are mostly determined by change in the polarisation angle rather than a change in the degree of polarisation. This is the reason

\footnotetext{
${ }^{6}$ In order to prevent the aliasing noise that effects power spectra with spectral index $\gtrsim 3$ when a Galactic cut is applied we used an apodysed Galactic cut off and the modified power spectra definition given in Giardino et al. (2001), in which the terms $a_{l 0}$ are set to zero.
}

why temperature fluctuations in the $E$ and $B$ components can become more intense than temperature fluctuations in the $T$ channel.

The figure shows that at $30 \mathrm{GHz}$ the high latitude synchrotron signal may dominate the cosmological polarised signal over the entire multipole range. The extrapolated signal at $30 \mathrm{GHz}$ is consistent with the current upper limit on linearly polarised signal of $10 \mu \mathrm{K}$ at the $7^{\circ}$ scale in the 26-36 GHz band (Keating et al. 2001) and with the upper limit of $16 \mu \mathrm{K}$ at degree scale in the $26-46 \mathrm{GHz}$ band (Netterfield et al. 1995). These upper limits also refer to regions of the sky at galactic latitudes $|b|>20^{\circ}$.

At $100 \mathrm{GHz}$ the synchrotron signal can make a significant contribution to the sky polarisation at large angular scale but it is significantly weaker than the CMB $E$ component at sub-degree angular scale. Therefore even using the most conservative assumptions one can conclude that the contribution of synchrotron polarisation to the sky signal will not hinder detection of the CMB $E$ component, if this is present at sub-degree angular scale at the intensity predicted by current cosmological models.

From the diagram the absence of the beam cut off in the spectra of the synthetic maps is noticeable. These are artificial maps and therefore they were generated at full resolution.

\subsection{Scope and limitations of the synthetic maps}

The synthetic full-sky maps of the Stokes parameters $T, Q$ and $U$ of Galactic synchrotron emission at 30 and $100 \mathrm{GHz}$ are a first attempt to model the full-sky synchrotron polarisation at high frequencies. The maps are needed as input for the simulation activity that is carried out in preparation for the Planck missions.

To study the effects of polarised foreground on MAP observations of the CMB, synthetic maps of polarised synchrotron emission have been generated by Kogut \& Hinshaw (2000). They also assumed the polarised signal to be proportional to the total intensity $T$, with the Stokes $Q$ and $U$ components given by Eq. (2). For the polarisation degree, $f$, they assumed a varying Gaussian field such that $\langle f\rangle=0.1$. They constructed the polarisation angle starting from Gaussian random fields, $x$ and $y$, with flat power spectra ${ }^{7}$, which lead to $E$ and $B$ components whose spectra have indices of $\alpha \simeq 0$. The polarisation maps were only generated for a fiducial frequency of $40 \mathrm{GHz}$.

We have constructed the synthetic maps by combining in a conservative way many of the observational data currently available on synchrotron total emission and polarisation. The maps that can be obtained at higher frequencies by extrapolating the total intensity map with the spectral index map are consistent with available observational upper limits on synchrotron emission and polarisation at frequencies higher than $10 \mathrm{GHz}$. There remain

\footnotetext{
7 The fields were then convolved with a Gaussian with varying $F W H M$, as the authors were interested in investigating the effect on CMB observations of the eventual presence of a coherence angle in polarised Galactic emission.
} 


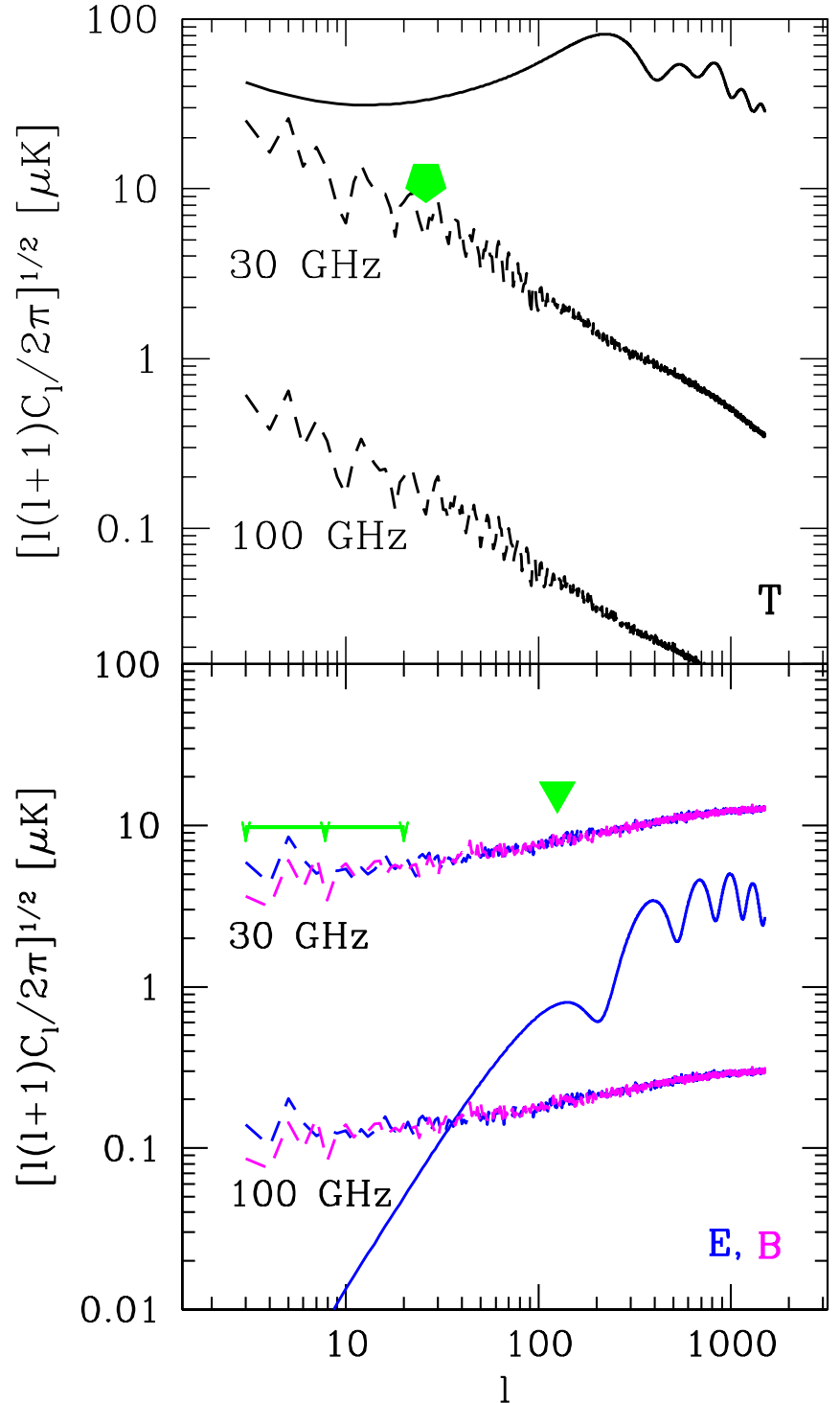

Fig. 11. The power spectra of the $T, E$ and $B$ components of the synthetic maps of Galactic synchrotron polarisation at 30 and $100 \mathrm{GHz}$ (dashed lines), for $|b|>20^{\circ}$, compared with the signal expected from the CMB (continuous line). The point with pentagonal shape indicates the COBE DMR upper limit to synchrotron temperature fluctuations at $31.5 \mathrm{GHz}$. The horizontal line is the upper limit to polarised signal by Keating et al. (2001) in the frequency band 26-36 GHz. The triangular point is the upper limit to polarised signal by Netterfield et al. (1995) in the frequency band 26-46 GHz.

however some inconsistencies between the synthetic polarisation maps extrapolated at $2.4 \mathrm{GHz}$ and the Parkes data.

The synthetic polarisation maps reproduce the slope of the angular power spectra of the $E$ and $B$ components of the Parkes survey, but not their amplitude at $2.4 \mathrm{GHz}$. Moreover, the power spectra of $T, E \times T$ and $L$ differ both in shape and normalisation to the ones of the Parkes data. This is because of the way that the toy model has been constructed. We derived information on the polarisation angle (and the $E$ and $B$ power spectra) from the Parkes survey, while for the intensity channel $T$ we used the data from the Haslam survey and for the polarisation fraction $f$ we assumed the theoretical value given by the synchrotron spectral index in a uniform magnetic field. The reasons behind these choices are the following.

As already mentioned, the Haslam map provides the best image of the total intensity of the full-sky synchrotron emission, to date. It is natural to adopt it as a template for $T$ as opposed to using the $T$ channel of the Parkes survey. This is limited in sky coverage and being at low Galactic latitude and a higher frequency contains a higher fraction of thermal emission from ionised gas.

The Parkes survey provides us with information about the statistical properties of the polarisation angle, so we adopt it for this purpose. Using the statistical properties of the polarisation angle derived from a survey at decimetric frequency is a conservative approach (i.e. it will unlikely lead to an underestimate of the level of fluctuation of polarised synchrotron emission). This is because Faraday rotation of a synchrotron background due to a foreground "screen" of ionised medium will tend to increase the level of fluctuations in the observed polarisation direction. On the other hand Faraday differential rotation may decrease the polarised fraction of the synchrotron signal at the Parkes frequency. Therefore using the amplitude of the $E$ and $B$ components of the Parkes data may lead to an underestimate of the polarisation fraction at higher frequencies $(\gtrsim 10 \mathrm{GHz})$.

Calculating the theoretical fraction of synchrotron polarisation by assuming a completely uniform magnetic fields could be considered an overly conservative approach because it is known that the interstellar medium is pervaded by an isotropic random ("turbulent") field. In the presence of a random magnetic field the observed level of polarisation is given by $f_{\mathrm{obs}}=d \cdot f$. Here $f$ is the intrinsic level of polarisation (given by Eq. (3)) and $d$ is a coefficient $(\leq 1)$ which depends on the relative intensity of the random field, $B_{\text {turb }}$, and the field which is regular in the whole resolution element, $B_{\text {reg }}$ (Burn 1966; Beck 1998).

Synchrotron polarisation observations at decimeter wavelength imply a ratio of regular to total field strengths within about a kpc from the Sun of $\left\langle B_{\mathrm{reg}} / B_{\mathrm{turb}}\right\rangle \simeq 0.6$ (Beck 2001), which, in the popular assumption of energy equipartition between cosmic rays and magnetic fields would imply an average level of depolarisation of $\langle d\rangle=0.3$. However the actual value varies substantially between points and may depend on the size of the resolution element. These values are derived from decimeter wavelength surveys with degree-scale resolution. The level of depolarisation by the random component of the magnetic field can be expected to decrease as the sky is observed at higher resolution and the projected size of the beam approaches the field coherence length. Indeed convolution of our synthetic maps with a Gaussian beam with a FWHM of $1^{\circ}$ yields an average level of depolarisation of $\langle d\rangle=0.5$. 


\section{Summary and conclusions}

In order to obtain information about the statistical properties of synchrotron polarised emission, we have derived the global angular power spectra of the Parkes survey, a radio continuum and polarisation survey of the Southern galactic plane at $2.4 \mathrm{GHz}$. The angular power spectrum of the polarised intensity $(L)$ is well approximated by a power law with fitted spectral index $\alpha_{L}=2.37 \pm 0.21$ in the multipole range $l=40-250$ that can be probed given the Parkes survey sky coverage and angular resolution. We exclude that up to multipole order $l=150$, the spectral index value is affected by the presence of discrete signals such us point sources or instrumental noise at pixel scale.

The angular power spectra of the $E$ and $B$ components of the polarised emission is significantly flatter than the spectrum of $L$. They have fitted spectral indices respectively of $\alpha_{E}=1.57 \pm 0.12$ and $\alpha_{B}=1.45 \pm 0.12$, in the multipole range $l=40-250$. This is because temperature variations in the Stokes channels $Q$ and $U$ of the data are mostly determined by variation in polarisation angle rather than polarisation intensity. The $E-B$ transform of the sinus and cosinus of the polarisation angle, the $e$ and $b$ components, are in fact also well described by power laws with fitted spectral indices of $\alpha_{e}=1.74 \pm 0.14$ and $\alpha_{b}=1.69 \pm 0.13$, in the same multipole range $l=40-250$.

We generated a full-sky map of a random polarisation angle having $e$ and $b$ components with power spectra with the same spectral indices of the $e$ and $b$ components of the Parkes data. We combined this map with a template of synchrotron emission at $408 \mathrm{MHz}$, derived from the $408-\mathrm{MHz}$ survey, and with a full-sky spectral index map, that we obtained by combining the $408-\mathrm{MHz}$ survey with the $1420-\mathrm{MHz}$ survey and the $2326-\mathrm{MHz}$ survey. The simple recipe on how to combine the three maps in order to derive a map of linear polarisation is given by Eq. (1) and Eq. (3).

In this way we have constructed synthetic maps of the Stokes parameters $(T, Q$ and $U$ ) of Galactic synchrotron emission at 30 and $100 \mathrm{GHz}$. The angular power spectra of the $E$ and $B$ components of these synthetic maps have slope very similar to the $E$ and $B$ components of the Parkes data. Their polarisation intensity is proportional to the total intensity $T$ as determined by the frequency spectral index in the conservative hypothesis of a uniform magnetic field.

We have compared the angular power spactra of the synthetic maps with predictions for the CMB and conclude that while direct observations of the CMB $E$ component at $30 \mathrm{GHz}$ may be precluded by synchrotron polarisation, at $100 \mathrm{GHz}$, this component of Galactic emission will not hinder the observation of cosmological polarisation, if this is present at the predicted levels.

The synthetic maps of the linear polarisation of Galactic synchrotron can be useful as a toy model to study the effect of the polarised foreground on planned observations of the CMB. Moreover they can serve as the basis for the development of more advanced models which incorpo- rate data on the structure of the Galactic magnetic field. In turn these refined models will be of fundamental importance in interpreting the microwave polarisation data that will be provided by the Planck and MAP satellite missions and the SPORT experiment.

The synthetic maps of the Stokes parameters $T$, $Q$ and $U$ of Galactic synchrotron emission at 30 and $100 \mathrm{GHz}$ together with the full-sky spectral index map are available for down loading at ftp://astro.esa.int/pub/synchrotron.

Acknowledgements. We thank D. P. Finkbeiner, M. Davis and D. Schlegel for providing us with the Cleaned $408 \mathrm{MHz}$ map, E. Hivon for helpful discussions, A. R. Duncan, R. F. Haynes, K. L. Jones and R. T. Stewart for making the Parkes survey publicly available and U. Seljak \& M. Zaldarriaga for their CMBFAST software, which was used to generate the CMB angular power spectra. The HEALPix analysis package (http://www.eso.org/science/healpix) was used extensively throughout this paper. We gratefully acknowledge very useful comments from an anonymous referee.

\section{References}

Baccigalupi, C., Burigana, C., Perrotta, F., et al. 2001, A\&A, 372,8

Banday, A. J., \& Wolfendale, A. W. 1990, MNRAS, 245, 182

Beck, R. 1998, Galactic Foreground Polarisation, Proceeding of a Workshop held in Bonn, ed. E. M. Berkhuijsen

Beck, R. 2001, Galactic and extragalactic magnetic fields, ed. R. Diehl et al., Space Science Reviews (Kluwer, Dordrecht)

Bennett, C. L., Smoot, G. F., Hinshaw, et al. 1992, ApJ, 396, L7

Beuermann, K., Kanbach, G., \& Berkhuijsen, E. M. 1985, A\&A, 153, 17

Bond, J. R., \& Efstathiou, G. 1987, MNRAS, 226, 655

Bouchet, F. R., \& Gispert, R. 1999, New Astron., 4, 443

Burn, B. J. 1966, MNRAS, 133, 67

Cortiglioni, S., \& Spoelstra, T. A. T. 1995, A\&A, 302, 1

Davies, R. D., \& Wilkinson, A. 1999, in Microwave Foregrounds, ASP Conf. Ser., 181, 77

de Oliveira-Costa, A., Tegmark, M., Page, L. A., \& Boughn, S. P. 1998, ApJ, 509, L9

Duncan, A. R., Haynes, R. F., Jones, K. L., \& Stewart, R. T. 1997, MNRAS, 291, 279

Duncan, A. R., Reich, P., Reich, W., \& Fürst, E. 1999, A\&A, 350,447

Fabbri, R., Cortiglioni, S., Cecchini, et al. 1999, in 3K cosmology, AIP Conf. Proc., 476, 194

Gaensler, B. M., Dickey, J. M., McClure-Griffiths, N. M., et al. 2001, ApJ, 549, 959

Giardino, G., Banday, A. J., Fosalba, P., et al. 2001, A\&A, 371,708

Górski, K. M., Hivon, E., \& Wandelt, B. D. 1999, in Proceedings of the MPA/ESO Cosmology Conference Evolution of Large-Scale Structure, ed. A. J. Banday, R. S. Sheth, \& L. Da Costa, 37

Gray, A. D., Landecker, T. L., Dewdney, P. E., et al. 1999, ApJ, 514, 221

Haslam, C. G. T., Salter, C. J., Stoffel, H., et al. 1982, A\&AS, 47,1

Hedman, M. M., Barkats, D., Gundersen, J. O., Staggs, S. T., \& Winstein, B. 2001, ApJ, 548, L111 
Jonas, J. L., Baart, E. E., \& Nicolson, G. D. 1998, MNRAS, Platania, P., Bensadoun, M., Bersanelli, M., et al. 1998, ApJ, 297, 977

Kamionkowski, M. 1997, Phys. Rev. D, 55, 7368

Keating, B. G., O'Dell, C. W., de Oliveira-Costa, A., et al. 2001, ApJ, 560, L1 505,473

Prunet, S., Sethi, S. K., Bouchet, F. R., et al. 1998, A\&A, 339, 187

Reich, P., \& Reich, W. 1986, A\&AS, 63, 205

Kogut, A., Banday, A. J., Bennett, C. L., et al. 1996, ApJ, 464, L5

Kogut, A., \& Hinshaw, G. 2000, ApJ, 543, 530

Kosowsky, A. 1996, Ann. Phys., 246, 49

Lawson, K. D., Mayer, C. J., Osborne, J. L., et al. 1987, MNRAS, 225, 307

Mandolesi, N., \& Puget, J.-L. 1998, AAO for Planck HFI and LFI (Paris: ESA)

Netterfield, C. B., Ade, P. A. R., Bock, J. J., et al. 2001, ApJ, submitted

Netterfield, C. B., Jarosik, N., Page, L., et al. 1995, ApJ, 445, L69

Rybicki, G. B., \& Lightman, A. P. 1979, Radiative Process in Astrophysics (Cambridge University Press)

Sokoloff, D. D., Bykov, A. A., Shukurov, A., et al. 1998, MNRAS, 299, 189

Tribble, P. C. 1991, MNRAS, 250, 726

Tucci, M., Carretti, E., Cecchini, S., et al. 2000, New Astr., 5, 181

Uyanıker, B., Fürst, E., Reich, W., Reich, P., \& Wielebinski, R. 1999, A\&AS, 138, 31

Wright, E. L. 1987, ApJ, 320, 818

Zaldarriaga, M. 2001, Phys. Rev. D, submitted

Zaldarriaga, M., \& Seljak, U. 1997, Phys. Rev. D, 55, 1830 